\title{
AVALIAÇÃO DO ESTADO NUTRICIONAL DE BANANEIRAS DO SUBGRUPO CAVENDISH NO ESTADO DE SÃO PAULO: NORMAS DRIS E NÍVEIS CRÍTICOS DE NUTRIENTES ${ }^{1}$
}

\author{
LUIZ ANTONIO JUNQUEIRA TEIXEIRA², FERNANDO CÉSAR BACHIEGA ZAMBROSI², \\ JOSÉ EMÍLIO BETTIOL NETO ${ }^{4}$
}

RESUMO - São apresentadas normas DRIS preliminares e derivados níveis críticos de nutrientes no tecido foliar (NC) para bananeiras do subgrupo Cavendish, nas condições de cultivo do Estado de São Paulo. As normas DRIS e os NCs foram obtidos a partir de um banco de dados com 188 registros, relacionando produtividade e teores foliares de N, P, K, Ca, Mg, B, Cu, Fe, Mn e $\mathrm{Zn}$ das cultivares Grande Naine e Nanicão. Esses registros foram extraídos de experimentos de adubação realizados no Planalto Paulista e no Vale do Ribeira, em áreas irrigadas e de sequeiro, durante cinco ciclos de cultivo, variando fontes e doses de fertilizantes. Mesmo com a grande diversidade nas condições de cultivo, a relação entre o índice de balanço nutricional e a produtividade das plantas foi altamente significativa $\left(\mathrm{R}^{2}=0,60 ; p<0,0001\right)$. O método para derivar os NCs a partir das normas DRIS foi eficiente, obtendo-se valores próximos daqueles citados na literatura, com exceção do $\mathrm{NC}$ para $\mathrm{K}$, que, para as condições locais, foi inferior.

Termos para indexação: adubação, análise foliar, banana, fruticultura, Musa spp., nutrição mineral.

\section{NUTRITIONAL DIAGNOSIS IN BANANA IN THE STATE OF SÃO PAULO (BRAZIL): DRIS NORMS AND CRITICAL LEVELS}

ABSTRACT - Preliminary DRIS norms and leaf nutrient critical levels (NCL) for Cavendish bananas growing in plantations of São Paulo State (Brazil) are presented. DRIS norms and NCL were established from a data bank of leaf nutrient concentration (N, P, K, Ca, $\mathrm{Mg}, \mathrm{B}, \mathrm{Cu}, \mathrm{Fe}, \mathrm{Mn}$ and $\mathrm{Zn}$ ) and yield of banana with 188 samples. Data bank comprises information from plants of Grand Naine and Giant Cavendish growing in irrigated and non-irrigated areas, with different sources and rates of fertilizers during five crop cycles. Despite of the great diversity of cropping conditions, the regression between nutrient balance index and fruit yield was significant $\left(\mathrm{R}^{2}=0.60 ; p<0.0001\right)$. The NCLs were derived using multiple linear regressions relating to the foliar nutrient concentration with DRIS indices of all nutrients. These values were similar to those presented in the literature, except the NCL for K which the value for local conditions was lower.

Index terms: Musa spp, plant analysis, mineral nutrition, fruit crops.

\section{INTRODUÇÃO}

O Estado de São Paulo é o maior produtor nacional de bananas, colhendo cerca de 1,1 milhão de toneladas de frutos/ ano em 55 mil hectares, dos quais aproximadamente $70 \%$ se encontram no Vale do Ribeira (IBGE, 2006). Destaca-se, também, a recente expansão da bananicultura para o Planalto Paulista, onde áreas com cultivos tradicionais (café, pastagem, etc.) têm na fruticultura alternativa de alta rentabilidade e que traz importantes benefícios sociais, como geração de empregos e manutenção de pequenos agricultores na zona rural.

O diagnóstico nutricional constitui-se num instrumento eficiente para detectar desequilíbrios e auxiliar no processo de recomendação de fertilizantes para bananeiras. Usualmente, os diagnósticos são feitos a partir da análise química do tecido foliar e posterior comparação com teores foliares ótimos (níveis críticos ou faixas de suficiência). Considera-se que nutrientes, cuja concentração esteja fora desses limites, provavelmente restrinjam o crescimento, o rendimento ou a qualidade dos frutos (Martin-
Prével, 1990; Lahav, 1995). Segundo Beaufils (1973) e Walworth \& Sumner (1987), uma abordagem alternativa, possivelmente mais eficiente para revelar desequilíbrios nutricionais e mais robusta em relação a variações na amostragem (idade da planta, época, etc.), seria o sistema integrado de diagnose e recomendação (DRIS), no qual são utilizadas relações entre as concentrações foliares dos nutrientes para interpretar os resultados de análise de tecido. O DRIS foi desenvolvido originalmente por Beaufils (1973), com propósitos amplos, visando a diagnosticar as causas primárias e secundárias que afetam a produtividade das culturas; no entanto, atualmente, o DRIS vem-se popularizando como um critério de avaliação do estado nutricional das plantas (Bataglia \& Santos, 1990).

O nível crítico (NC) para o teor de um nutriente corresponde a sua concentração foliar requerida para a obtenção de crescimento, produção e/ou qualidade ótima, assumindo que não existem outros fatores limitantes. $\mathrm{O}$ emprego desse padrão no diagnóstico nutricional requer cuidados, pois, além da amostragem padronizada para bananeira, devem-se considerar possíveis

(Trabalho 177-06). Recebido em : 09-11-2006. Aceito para publicação em : 06-09-2007

2 Pesquisador do Centro de Solos e Recursos Ambientais - IAC/APTA, Cx. Postal 28, 13012-970, Campinas, SP, teixeira@iac.sp.gov.br

Pesquisador do Centro de Solos e Recursos Ambientais - IAC/APTA. zambrosi@iac.sp.gov.br

${ }^{4}$ Pesquisador do Centro de Fruticultura - IAC/APTA. bettiolneto@iac.sp.gov.br 
variações devidas ao ambiente e às cultivares (Martin-Prével, 1977; Lahav \& Turner, 1985; Turner \& Lahav, 1985).

Segundo Needham et al. (1990), tão importante quanto atingir produtividade ótima na determinação de padrões para o diagnóstico nutricional é a condição de que todos os outros fatores de produção não sejam limitantes, situação que exige experimentos controlados, o que nem sempre é obtido. O DRIS, além de ferramenta para diagnóstico, possibilita estimar níveis críticos no tecido foliar para cada nutriente em condições de equilíbrio nutricional, como demonstraram Needham et al. (1990) e Joseph \& Ranganathan (1996).

Angeles et al. (1993), ao analisarem resultados de experimentos de adubação em bananeira, constataram limitações em diagnósticos nutricionais estabelecidos por nutrientes individualmente (níveis críticos ou faixas de suficiência), sem considerar as relações de proporção entre eles. Assim, a partir de um banco de dados com informações obtidas em experimentos realizados em diversas condições de ambiente e manejo da cultura, os referidos autores propuseram padrões (normas) DRIS preliminares para essa cultura e recomendaram que essas normas fossem validadas.Por serem normas preliminares, Angeles et al. (1993) recomendaram em experimentos de adubação para subsidiar possíveis ajustes. No Brasil, Silva (2004) gerou normas DRIS para bananeiras 'Prata Anã' cultivada sob irrigação, no norte do Estado de Minas Gerais. Empregando essas normas DRIS, Silva \& Carvalho (2005) avaliaram o estado nutricional de bananais de 'Prata-Anã', mas não sem obtenção de iveram correlação significativa entre o índice de balanço nutricional (IBN) e a produtividade, indicando, segundo os autores, que outros fatores de ordem não-nutricional estavamriam limitando a produção. Teixeira et al. (2002b), ao avaliaraem o desempenho de diagnósticos nutricionais (NC e do DRIS) para N e K, para 'Nanicão', concluíram que o DRIS foi mais eficiente para diagnosticar deficiências de N; enquanto, para $\mathrm{K}$, o DRIS e o critério de NC apresentaram desempenhos semelhantes, tanto no cultivo em condições de sequeiro como sob irrigação.

Este trabalho visa a propor normas DRIS preliminares e derivar níveis críticos de nutrientes no tecido foliar para bananeiras do subgrupo Cavendish nas condições de cultivo do Estado de São Paulo.

\section{MATERIAL E MÉTODOS}

As normas para sistema integrado de diagnose e recomendação (DRIS) e os níveis críticos de nutrientes no tecido foliar (NC) foram obtidos a partir de um banco de dados com 188 registros, relacionando produtividade e teores foliares de N, P, K, $\mathrm{Ca}, \mathrm{Mg}, \mathrm{B}, \mathrm{Cu}, \mathrm{Fe}, \mathrm{Mn}$ e $\mathrm{Zn}$ em bananais com as cultivares Grande Naine e Nanicão, ambas pertencentes ao grupo genômico AAA e subgrupo Cavendish. Esses registros foram coletados em experimentos de adubação realizados no Planalto Paulista (Latossolo Vermelho eutroférrico) e no Vale do Ribeira (Latossolo Amarelo distrófico), em áreas irrigadas e de sequeiro, com variação nas fontes (orgânica e mineral) e doses de fertilizantes, durante cinco ciclos de cultivo,.

O tecido foliar foi amostrado de acordo com as recomendações de Martin-Prével (1984). A amostragem consistiu na coleta da porção central do limbo da terceira folha, contandose a partir do ápice, na época em que a inflorescência apresentava duas ou três pencas masculinas abertas. As amostras foram processadas e analisadas quanto aos teores de teores foliares de macro (N, P, K, Ca e Mg) e de micronutrientes (B, Cu, Fe, Mn e Zn) de acordo com Bataglia et al. (1983). A produção foi estimada por meio de pesagem dos cachos no ponto de colheita comercial para o mercado interno.

As normas para o sistema integrado de diagnose e recomendação (DRIS) e seus desvios foram calculados de acordo com Walworth \& Sumner (1987). O conjunto de dados foi separado em duas sub-populações $(\mathrm{A}=$ alta, e $\mathrm{B}=$ baixa produtividade), sendo $45 \mathrm{t}$ ha-1 por ciclo, a produtividade que as discriminou.

As funções intermediárias para a geração dos índices DRIS foram calculadas pela fórmula proposta por Jones (1981):

$f(X / Y)=(X / Y-x / y) \cdot K / s$

na qual:

$\mathbf{X} / \mathbf{Y}=$ valor da relação entre as concentrações dos dois nutrientes na planta sob diagnóstico;

$\mathbf{x} / \mathbf{y}=$ valor ótimo (norma) para a relação entre os nutrientes;

$\mathbf{K}=10$ (constante de valor arbitrário), e

$\mathbf{s}=$ desvio-padrão da relação x/y (norma) na população de referência.

Os índices DRIS para cada nutriente (NI, PI, KI, CaI, MgI, $\mathrm{BI}, \mathrm{CuI}$, FeI, MnI e ZnI) foram determinados segundo Walworth \& Sumner (1987). O índice de balanço nutricional (IBN) foi calculado somando-se os valores em módulo obtidos para cada nutriente:

$$
\begin{aligned}
& \mathbf{I B N}=|\mathbf{N I}|+|\mathbf{P I}|+|\mathbf{K I}|+|\mathbf{C a I}|+|\mathbf{M g I}|+|\mathbf{B I}|+|\mathbf{C u I}|+|\mathbf{F e I}|+ \\
& |\mathbf{M n I}|+|\mathbf{Z n I}|
\end{aligned}
$$

Por fim, a relação entre o IBN e a produtividade dos bananais foi modelada por meio do ajuste de uma função matemática. Calculou-se, também, o coeficiente de determinação $\left(\mathrm{R}^{2}\right)$ do modelo, o qual representa, em porcentagem, quanto da variação da produtividade dos bananais pode ser explicada pela relação ajustada.

Teores de N, P, K, Ca, Mg, B, Cu, Fe, Mn e Zn foliares ideais para bananeira foram estimados por meio de regressão múltipla entre as concentrações na folha diagnóstico e índices DRIS para todos os nutrientes. Seguindo-se as recomendações de Joseph \& Ranganathan (1996), optou-se pelo emprego de regressões múltiplas, pois, desse modo, são consideradas as possíveis interações entre nutrientes na determinação dos teores ideais.

No modelo de regressão múltipla, tem-se que:

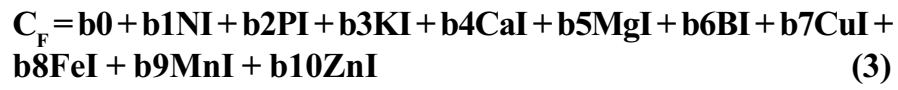


no qual:

$\mathbf{C}^{\mathbf{F}}=$ concentração foliar de um nutriente em particular; NI, PI, KI, CaI, MgI, BI, CuI , FeI, MnI e ZnI = índices DRIS.

Os índices DRIS (NI, PI, KI, CaI, MgI, BI, CuI, FeI, MnI e $\mathrm{ZnI)} \mathrm{não} \mathrm{são} \mathrm{independentes,} \mathrm{pois} \mathrm{apresentam} \mathrm{a} \mathrm{seguinte} \mathrm{relação:}$

\section{$\mathbf{N I}+\mathbf{P I}+\mathbf{K I}+\mathbf{C a I}+\mathbf{M g I}+\mathbf{B I}+\mathbf{C u I}+\mathbf{F e} I+\mathrm{MnI}+\mathbf{Z n I}=\mathbf{0}$}

Assim, na equação 3, o número de variáveis independentes da regressão múltipla pode ser reduzido, empregando-se a relação descrita na equação 4 , obtendo-se o seguinte modelo reduzido:

$\mathrm{C}_{\mathrm{F}}=\mathrm{b} 0+\mathrm{b} 1 \mathrm{X} 1+\mathrm{b} 2 \mathrm{X2}+\mathrm{b3X3}+\mathrm{b} 4 \mathrm{X} 4+\mathrm{b5} \times 5+\mathrm{b} 6 \mathrm{X6}+\mathrm{b} 7 \mathrm{X} 7+$
$\mathrm{b8X8}+\mathrm{b} 9 \mathrm{X} 9$

na qual:

$\mathbf{C}_{\mathbf{F}}=$ concentração foliar de um nutriente em particular;

$\mathbf{X}_{1}$ a $\mathbf{X}_{9}=$ índices DRIS para nove nutrientes selecionados.

Nesse modelo, somente os nove índices DRIS que apresentaram as maiores correlações com as concentrações foliares $\left(\mathrm{C}_{\mathrm{F}}\right)$, foram selecionados para a regressão. Posteriormente, foram ajustadas regressões seguindo o modelo reduzido (equação 5). As concentrações foliares de N, P, K, Ca, Mg, B, Cu, Fe, Mn e $\mathrm{Zn}$ ótimas para bananeira foram obtidas igualando-se todos os índices a zero na equação reduzida (equação 5), pois, teoricamente, quando todos esses índices tendem a zero, tem-se a condição ótima de equilíbrio nutricional da planta. Por fim, foram associados intervalos de confiança (IC 99\%) às estimativas dos teores ótimos de $\mathrm{N}, \mathrm{P}, \mathrm{K}, \mathrm{Ca}, \mathrm{Mg}, \mathrm{B}, \mathrm{Cu}, \mathrm{Fe}, \mathrm{Mn}$ e $\mathrm{Zn}$ para bananeira gerados a partir das normas DRIS.

\section{RESULTADOS E DISCUSSÃO}

$\mathrm{Na}$ Tabela 1, encontram-se as médias, amplitudes e desvios-padrão dos teores foliares de $\mathrm{N}, \mathrm{P}, \mathrm{K}, \mathrm{Ca}, \mathrm{Mg}, \mathrm{B}, \mathrm{Cu}, \mathrm{Fe}$, $\mathrm{Mn}$ e $\mathrm{Zn}$ e da produtividade das bananeiras obtidas a partir do banco de dados com 188 observações. As médias dos teores foliares apresentaram ampla variação em torno dos valores de níveis críticos (NC) citados por Lahav (1995) (Tabela 2), indicando elevada diversidade no estado nutricional das plantas, o que, de certo modo, refletiu-se na variação da produtividade. Essas variações são decorrentes das diferentes condições de cultivo (ou nutricionais) das plantas que formaram o banco de dados. Segundo Mourão Filho (2004), a qualidade de normas DRIS não se relaciona diretamente com o tamanho do banco de dados; independentemente da quantidade de registros, a qualidade das observações deve ser o objetivo maior. Para formar o banco de dados deste trabalho, todos os 188 registros foram coletados em condições controladas, pois provieram de áreas experimentais.

Os teores foliares das populações de alta e de baixa produtividade são apresentados na Tabela 3. Observaram-se diferenças estatisticamente significativas entre as populações de alta e baixa produtividade, o que é um bom indicativo sobre a confiabilidade das normas DRIS a serem geradas (Reis Júnior \& Monnerat, 2003). As concentrações médias de N, P, K, Cu e Zn foram significativamente maiores nas amostras provenientes de populações de alta produtividade. Nitrogênio e potássio são os nutrientes de maior acumulação na biomassa das bananeiras (Lahav, 1995), enquanto o zinco é o micronutriente cuja deficiência é a mais comum nos bananais (Lahav, 1995; Silva \& Rodrigues, 2001).

As razões entre teores foliares, calculadas para as populações de alta e de baixa produtividade, encontram-se Tabela 4. De acordo com o teste F, que compara as variâncias entre as populações de alta e de baixa produtividade, as razões entre os teores serviram para discriminar essas duas populações, pois, das 45 razões calculadas, apenas quatro não tiveram variâncias significativamente diferentes entre as duas populações. As normas DRIS (médias das relações, desvios e coeficientes de variação) preliminares, geradas a partir da população de alta produtividade, que poderão ser utilizadas como referência na diagnose nutricional nas regiões estudadas, estão apresentadas na Tabela 5. A partir das referidas normas, foram calculados os índices DRIS para cada nutriente, seguindo o método proposto por Jones (1981).

A regressão entre o IBN e a produtividade (Figura 1) revelou que a produção de frutos esteve significativamente associada ao estado nutricional das plantas $\left(\mathrm{R}^{2}=0,60, p<0,0001\right)$. Valores de IBN baixos são indicadores de plantas bem nutridas $\mathrm{e}$ corresponderam às amostras de plantas com maior produtividade, ratificando a importância do balanço nutricional para a produção de frutos. Silva \& Carvalho (2005) não obtiveram correlação significativa entre IBN e produtividade em bananeira 'Prata- Anã' no Norte de Minas Gerais, atribuindo as variações observadas a fatores não-nutricionais. Entretanto, esse índice tem sido eficiente para demonstrar o efeito do balanço nutricional das plantas sobre a produtividade para várias espécies frutíferas, como apresentado por Mourão Filho(2004)

A estimativa de teores foliares ótimos para a bananeira, a partir dos resultados do DRIS, foi obtida assumindo-se que plantas com índices DRIS tendendo a zero, estejam bem nutridas. Assim, equações de regressão múltipla foram ajustadas, relacionandose teores foliares com índices DRIS. Foram selecionados para a equação de cada nutriente, os nove índices DRIS que apresentaram maior correlação com os teores foliares do mesmo Tabela 6), gerando as equações apresentadas na Tabela 7, todas elas .com ajustes altamente significativos $(p<0,001)$. As concentrações foliares ótimas para bananeira foram obtidas igualando-se todos os índices a zero em cada equação. Esses teores são apresentados na Tabela 2, juntamente com os padrões comumente empregados na diagnose nutricional da cultura.

Os valores gerados a partir de normas DRIS locais e os apresentados na literatura foram bastante semelhantes (Tabela 2), o que indica que este método para a obtenção de teores ótimos foi eficiente. Entre os macronutrientes, a maior diferença entre os padrões da literatura e o teor estimado a partir do DRIS foi em relação ao teor de K. É possível que os padrões para K, apresentados na literatura, sejam superestimados para as condições dos bananais do Estado de São Paulo, visto que, em 
experimentos- com adubação adequada e com plantas apresentando produtividades elevadas, não foi possível atingilos (Teixeira et al., 2002a; Damatto Jr., 2005; Teixeira et al., 2006). Em relação aos micronutrientes, exceto para o zinco, todos os teores derivados dos índices DRIS estavam contidos nas suas respectivas faixas de suficiência propostas por Quaggio \& Raij (1997), indicando que as mesmas, apesar de amplas, compreendem os teores adequados dos micronutrientes.

TABELA 1- Estatísticas do banco de dados com 188 registros de teores foliares de N, P, K, Ca, Mg, B, Cu, Fe, Mn e $\mathrm{Zn}$ e produtividade de bananeira do subgrupo Cavendish. Estado de São Paulo, 2007.

\begin{tabular}{|c|c|c|c|c|}
\hline Variável & Média & Mínimo & Máximo & $\begin{array}{l}\text { Desvio- } \\
\text { padrão }\end{array}$ \\
\hline & \multicolumn{4}{|c|}{--------------------- $\mathrm{g} \mathrm{kg}^{-1}$} \\
\hline $\mathbf{N}$ & 26,8 & 17,2 & 40,0 & 5,10 \\
\hline $\mathbf{P}$ & 1,6 & 0,7 & 2,4 & 0,30 \\
\hline $\mathbf{K}$ & 25,2 & 4,1 & 38,9 & 6,48 \\
\hline $\mathbf{C a}$ & 12,0 & 3,1 & 25,0 & 4,52 \\
\hline Mg & 3,8 & 2,1 & 6,4 & 1,15 \\
\hline $\mathbf{B}$ & 19 & 10 & 38 & 6,4 \\
\hline $\mathbf{C u}$ & 8 & 4 & 17 & 2,6 \\
\hline $\mathbf{F e}$ & 87 & 35 & 419 & 46,5 \\
\hline Mn & 590 & 80 & 2160 & 405,1 \\
\hline \multirow[t]{2}{*}{$\mathbf{Z n}$} & 15 & 9 & 29 & 4,7 \\
\hline & 260 & ------- & & \\
\hline ?rodutividade & 36,0 & 9,8 & 66,1 & 11,0 \\
\hline
\end{tabular}

Recomenda-se que as normas preliminares apresentadas neste trabalho sejam validadas em condições de cultivo comerciais e por meio de experimentos de adubação, especialmente fatoriais, como assinalado por Beverly \& Hallmark (1992) e Angeles et al. (1993). Outrossim, a obtenção de normas DRIS confiáveis e de aplicabilidade mais ampla será alcançada na medida em que novos registros de campo forem agregados ao banco de dados.

TABELA 2 - Teores foliares de N, P, K, Ca, Mg, B, Cu, Fe, Mn e $\mathrm{Zn}$ ótimos para bananeira, que são comumente empregados na diagnose nutricional, e os teores derivados dos índices DRIS para subgrupo Cavendish. Estado de São Paulo, 2007.

\begin{tabular}{|c|c|c|c|}
\hline Nutriente & Lahav (1995) & IAC-BT 100 ${ }^{(1)}$ & $\begin{array}{c}\text { Derivados do } \\
\text { DRIS }^{(2)}\end{array}$ \\
\hline & \multicolumn{3}{|c|}{------------------------ g kg $^{-1}$} \\
\hline $\mathbf{N}$ & 26 & $27-36$ & $28,5 \pm 0,9$ \\
\hline $\mathbf{P}$ & 2 & $1,8-2,7$ & $1,7 \pm 0,05$ \\
\hline $\mathbf{K}$ & 30 & $35-54$ & $27,9 \pm 0,9$ \\
\hline $\mathbf{C a}$ & 5 & $3-12$ & $10,8 \pm 0,4$ \\
\hline \multirow[t]{2}{*}{ Mg } & 3 & $3-6$ & $3,6 \pm 0,1$ \\
\hline & & $--\mathrm{mg} \mathrm{kg}^{-1}$ & 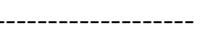 \\
\hline B & 11 & $10-25$ & $17,7 \pm 0,6$ \\
\hline $\mathbf{C u}$ & 9 & $6-30$ & $9,5 \pm 0,4$ \\
\hline $\mathbf{F e}$ & 80 & $80-360$ & $87,3 \pm 5,0$ \\
\hline Mn & 25 & $200-2000$ & $436,5 \pm 16,6$ \\
\hline $\mathbf{Z n}$ & 18 & $20-50$ & $17,3 \pm 0,6$ \\
\hline
\end{tabular}

(1) Quaggio \& Raij (1997); (2) Intervalo de confiança (IC99\%) para teor foliar ótimo derivado por meio de regressão múltipla entre teores foliares e índices DRIS.

TABELA 3 - Valores médios dos Tteores foliares de nutrientes e da produtividade da bananeira do subgrupo Cavendish, observadas em populações de alta (A) e baixa (B) produtividade. Estado de São Paulo, 2007.

\begin{tabular}{|c|c|c|c|c|c|}
\hline \multirow[t]{2}{*}{ Forma de expressão } & \multicolumn{2}{|c|}{$\begin{array}{l}\text { População de alta } \\
\text { produtividade (A) }\end{array}$} & \multicolumn{2}{|c|}{$\begin{array}{l}\text { População de baixa produtividade } \\
\qquad(\mathrm{B})^{(2)}\end{array}$} & \multirow{2}{*}{$\begin{array}{c}\text { Teste t para médias } \\
(\mathrm{M}) \\
\text { Valor } \mathrm{p}^{(4)}\end{array}$} \\
\hline & Média & $\mathrm{DP}^{(3)}$ & Média & DP & \\
\hline $\mathrm{N}\left(\mathrm{gkg}^{-1}\right)$ & 29,3 & 5,79 & 26,0 & 4,65 & 0,001 \\
\hline$P\left(\mathrm{~g} \mathrm{~kg}^{-1}\right)$ & 1,7 & 0,33 & 1,5 & 0,27 & $<0,001$ \\
\hline $\mathrm{K}\left(\mathrm{g} \mathrm{kg}^{-1}\right)$ & 28,4 & 4,68 & 24,3 & 6,65 & $<0,001$ \\
\hline $\mathrm{Ca}\left(\mathrm{g} \mathrm{kg}^{-1}\right)$ & 11,2 & 4,18 & 12,2 & 4,61 & 0,187 \\
\hline $\operatorname{Mg}\left(\mathrm{gkg}^{-1}\right)$ & 3,7 & 1,16 & 3,8 & 1,14 & 0,546 \\
\hline $\mathrm{B}\left(\mathrm{mgkg}^{-1}\right)$ & 18 & 5,08 & 20 & 6,7 & 0,097 \\
\hline $\mathrm{Cu}\left(\mathrm{mg} \mathrm{kg}^{-1}\right)$ & 10 & 3,02 & 8 & 2,3 & $<0,001$ \\
\hline $\mathrm{Fe}\left(\mathrm{mgkg}^{-1}\right)$ & 91 & 41,17 & 86 & 48,0 & 0,555 \\
\hline $\operatorname{Mn}\left(\mathrm{mgkg}^{-1}\right)$ & 444 & 223,29 & 632 & 435,4 & $<0,001$ \\
\hline $\mathrm{Zn}\left(\mathrm{mg} \mathrm{kg}^{-1}\right)$ & 18 & 5,05 & 14 & 4,3 & $<0,001$ \\
\hline Produtividade $(\mathrm{t} / \mathrm{ha})$ & 50 & 5,0 & 32 & 8,4 & $<0,001$ \\
\hline
\end{tabular}

(1) Produtividade $>45 \mathrm{t} \mathrm{ha}^{-1}$; (2) produtividade $<45 \mathrm{tha}^{-1}$; (3) desvio-padrão; (4) probabilidade do erro tipo I associada ao teste t ou nível de significância do teste; valores em negrito são significativos a $5 \%$ de probabilidade $(p<0,05)$. 
TABELA 4 - Razões entre teores foliares; teste t para diferença entre médias e teste $F$ para razão entre variâncias observadas em populações de alta (A) e baixa (B) produtividade de bananeira Cavendish. Estado de São Paulo, 2007.

\begin{tabular}{|c|c|c|c|c|c|c|}
\hline \multirow[t]{2}{*}{ Forma de expressão } & \multicolumn{2}{|c|}{$\begin{array}{c}\text { População de alta } \\
\text { produtividade }(A)^{(1)}\end{array}$} & \multicolumn{2}{|c|}{$\begin{array}{l}\text { População de baixa } \\
\text { produtividade }(B)^{(2)}\end{array}$} & \multirow{2}{*}{$\begin{array}{l}\frac{\text { Teste t para }}{\text { médias }(M)} \\
\text { Valor } p^{(4)}\end{array}$} & \multirow{2}{*}{$\begin{array}{c}\text { Teste F para } \\
\text { variâncias }(S) \\
\text { Valor } p^{(5)}\end{array}$} \\
\hline & Média & $\mathbf{D P}^{(3)}$ & Média & DP & & \\
\hline $\mathbf{P} / \mathbf{N}$ & 0,06 & 0,005 & 0,06 & 0,011 & 0,628 & $<0,001$ \\
\hline $\mathbf{N} / \mathbf{K}$ & 1,05 & 0,204 & 1,19 & 0,585 & 0,013 & $<0,001$ \\
\hline $\mathbf{P} / \mathbf{K}$ & 0,06 & 0,011 & 0,07 & 0,026 & 0,054 & $<0,001$ \\
\hline $\mathrm{Ca} / \mathbf{N}$ & 0,38 & 0,091 & 0,48 & 0,183 & $<0,001$ & $<0,001$ \\
\hline $\mathbf{M g} / \mathbf{N}$ & 0,12 & 0,021 & 0,15 & 0,042 & $<0,001$ & $<0,001$ \\
\hline $\mathbf{C a} / \mathbf{P}$ & 6,35 & 1,521 & 8,41 & 3,845 & $<0,001$ & $<0,001$ \\
\hline $\mathbf{M g} / \mathbf{P}$ & 2,10 & 0,354 & 2,61 & 0,959 & $<0,001$ & $<0,001$ \\
\hline $\mathbf{C a} / \mathbf{K}$ & 0,40 & 0,150 & 0,62 & 0,588 & $<0,001$ & $<0,001$ \\
\hline $\mathbf{M g} / \mathrm{K}$ & 0,13 & 0,042 & 0,19 & 0,159 & $<0,001$ & $<0,001$ \\
\hline $\mathrm{Ca} / \mathrm{Mg}$ & 3,01 & 0,425 & 3,16 & 0,577 & 0,078 & 0,025 \\
\hline $\mathbf{B} / \mathbf{N}$ & 0,64 & 0,172 & 0,78 & 0,276 & $<0,001$ & $<0,001$ \\
\hline $\mathbf{B} / \mathbf{P}$ & 10,72 & 2,916 & 13,64 & 5,510 & $<0,001$ & $<0,001$ \\
\hline $\mathbf{B} / \mathbf{K}$ & 0,65 & 0,146 & 0,98 & 0,879 & $<0,001$ & $<0,001$ \\
\hline $\mathrm{B} / \mathrm{Ca}$ & 1,86 & 0,877 & 1,83 & 0,917 & 0,853 & 0,760 \\
\hline $\mathrm{B} / \mathrm{Mg}$ & 5,34 & 1,900 & 5,45 & 1,873 & 0,740 & 0,874 \\
\hline $\mathrm{B} / \mathrm{Cu}$ & 1,99 & 0,692 & 2,78 & 1,169 & $<0,001$ & $<0,001$ \\
\hline $\mathrm{Fe} / \mathrm{B}$ & 5,32 & 2,931 & 4,71 & 3,159 & 0,246 & 0,589 \\
\hline Mn/B & 27,51 & 16,621 & 35,69 & 25,842 & 0,016 & 0,001 \\
\hline $\mathbf{B} / \mathbf{Z n}$ & 1,11 & 0,402 & 1,54 & 0,754 & $<0,001$ & $<0,001$ \\
\hline $\mathrm{N} / \mathrm{Cu}$ & 3,07 & 0,495 & 3,53 & 0,635 & $<0,001$ & 0,065 \\
\hline $\mathrm{P} / \mathrm{Cu}$ & 0,18 & 0,024 & 0,21 & 0,044 & $<0,001$ & $<0,001$ \\
\hline $\mathrm{Cu} / \mathrm{K}$ & 0,35 & 0,103 & 0,34 & 0,169 & 0,675 & $<0,001$ \\
\hline $\mathrm{Ca} / \mathrm{Cu}$ & 1,13 & 0,215 & 1,68 & 0,749 & $<0,001$ & $<0,001$ \\
\hline $\mathrm{Mg} / \mathrm{Cu}$ & 0,38 & 0,054 & 0,52 & 0,179 & $<\mathbf{0 , 0 0 1}$ & $<0,001$ \\
\hline $\mathrm{Fe} / \mathrm{Cu}$ & 8,97 & 2,363 & 11,68 & 7,001 & $<0,001$ & $<0,001$ \\
\hline $\mathrm{Mn} / \mathrm{Cu}$ & 49,49 & 31,852 & 88,96 & 66,078 & $<0,001$ & $<0,001$ \\
\hline $\mathrm{Zn} / \mathrm{Cu}$ & 1,81 & 0,248 & 1,93 & 0,588 & 0,070 & $<0,001$ \\
\hline $\mathrm{Fe} / \mathbf{N}$ & 2,99 & 0,920 & 3,30 & 1,539 & 0,109 & $<0,001$ \\
\hline $\mathrm{Fe} / \mathrm{P}$ & 50,43 & 15,909 & 59,02 & 42,919 & 0,048 & $<0,001$ \\
\hline $\mathrm{Fe} / \mathrm{K}$ & 3,23 & 1,454 & 4,10 & 3,546 & 0,020 & $<0,001$ \\
\hline $\mathrm{Fe} / \mathrm{Ca}$ & 8,10 & 2,280 & 8,07 & 9,092 & 0,972 & $<0,001$ \\
\hline $\mathrm{Fe} / \mathrm{Mg}$ & 23,93 & 5,707 & 22,97 & 12,746 & 0,487 & $<0,001$ \\
\hline $\mathrm{Fe} / \mathrm{Mn}$ & 0,28 & 0,196 & 0,24 & 0,341 & 0,424 & $<0,001$ \\
\hline $\mathrm{Fe} / \mathrm{Zn}$ & 4,98 & 1,229 & 6,23 & 2,709 & $<0,001$ & $<0,001$ \\
\hline $\mathbf{M n} / \mathbf{N}$ & 16,36 & 9,928 & 25,03 & 16,899 & $<0,001$ & $<0,001$ \\
\hline $\mathbf{M n} / \mathbf{P}$ & 273,98 & 168,757 & 442,44 & 321,109 & $<0,001$ & $<0,001$ \\
\hline $\mathbf{M n} / \mathbf{K}$ & 16,61 & 9,731 & 29,72 & 23,404 & $<0,001$ & $<0,001$ \\
\hline $\mathrm{Mn} / \mathrm{Ca}$ & 44,08 & 28,813 & 56,73 & 44,015 & $\mathbf{0 , 0 3 0}$ & 0,002 \\
\hline $\mathbf{M n} / \mathbf{M g}$ & 135,62 & 94,927 & 178,57 & 139,917 & 0,024 & 0,005 \\
\hline $\mathbf{M n} / \mathbf{Z n}$ & 27,96 & 19,554 & 49,50 & 36,906 & $<0,001$ & $<0,001$ \\
\hline $\mathbf{N} / \mathbf{Z n}$ & 1,71 & 0,287 & 1,93 & 0,460 & $<0,001$ & $<0,001$ \\
\hline $\mathbf{Z n} / \mathbf{P}$ & 10,07 & 1,436 & 9,39 & 2,335 & 0,023 & $<0,001$ \\
\hline $\mathbf{Z n} / \mathbf{K}$ & 0,63 & 0,177 & 0,64 & 0,329 & 0,948 & $<0,001$ \\
\hline $\mathrm{Ca} / \mathrm{Zn}$ & 0,63 & 0,132 & 0,94 & 0,494 & $<0,001$ & $<0,001$ \\
\hline $\mathrm{Mg} / \mathrm{Zn}$ & 0,21 & 0,031 & 0,29 & 0,122 & $<0,001$ & $<0,001$ \\
\hline
\end{tabular}

(1) Produtividade $>45 \mathrm{t} \mathrm{ha}^{-1}$; (2) produtividade $<45 \mathrm{t} \mathrm{ha}^{-1}$; (3) desvio-padrão; (4) probabilidade do erro tipo I associada ao teste $\mathrm{t}$ ou nível de significância do teste; ${ }^{(5)}$ probabilidade do erro tipo I associada ao teste $\mathrm{F}$ ou nível de significância do teste de comparação das variâncias da população de alta e baixa produtividade $\left(\mathrm{F}=\mathrm{S}_{\mathrm{B}} / \mathrm{S}_{\mathrm{A}}\right)$; valores em negrito são significativos a $5 \%$ de probabilidade $(p<0,05)$. 
TABELA 5 - Normas DRIS para bananeira Cavendish propostas a partir do banco de dados locais. Estado de São Paulo, 2007.

\begin{tabular}{|c|c|c|c|c|c|c|c|}
\hline $\begin{array}{l}\text { Forma de } \\
\text { expressão }\end{array}$ & Média & $\mathbf{s}^{(\mathbf{1})}$ & $C V^{(2)}$ & $\begin{array}{l}\text { Forma de } \\
\text { expressão }\end{array}$ & Média & $\mathbf{s}^{(\mathbf{1})}$ & $C V^{(2)}$ \\
\hline $\mathbf{P} / \mathbf{N}$ & 0,06 & 0,005 & 8,9 & $\mathrm{Mg} / \mathrm{Cu}$ & 0,38 & 0,054 & 14,3 \\
\hline $\mathbf{N} / \mathbf{K}$ & 1,05 & 0,204 & 19,5 & $\mathrm{Fe} / \mathrm{Cu}$ & 8,97 & 2,363 & 26,4 \\
\hline $\mathbf{P} / \mathbf{K}$ & 0,06 & 0,011 & 17,9 & $\mathbf{M n} / \mathbf{C u}$ & 49,49 & 31,852 & 64,4 \\
\hline $\mathbf{C a} / \mathbf{N}$ & 0,38 & 0,091 & 24,0 & $\mathrm{Zn} / \mathbf{C u}$ & 1,81 & 0,248 & 13,7 \\
\hline $\mathbf{M g} / \mathbf{N}$ & 0,12 & 0,021 & 16,6 & $\mathrm{Fe} / \mathbf{N}$ & 2,99 & 0,920 & 30,8 \\
\hline $\mathrm{Ca} / \mathrm{P}$ & 6,35 & 1,521 & 24,0 & $\mathrm{Fe} / \mathrm{P}$ & 50,43 & 15,909 & 31,5 \\
\hline $\mathbf{M g} / \mathbf{P}$ & 2,10 & 0,354 & 16,9 & $\mathrm{Fe} / \mathrm{K}$ & 3,23 & 1,454 & 45,0 \\
\hline $\mathrm{Ca} / \mathrm{K}$ & 0,40 & 0,150 & 37,3 & $\mathrm{Fe} / \mathrm{Ca}$ & 8,10 & 2,280 & 28,1 \\
\hline $\mathbf{M g} / \mathbf{K}$ & 0,13 & 0,042 & 31,5 & $\mathrm{Fe} / \mathrm{Mg}$ & 23,93 & 5,707 & 23,9 \\
\hline $\mathrm{Ca} / \mathrm{Mg}$ & 3,01 & 0,425 & 14,1 & $\mathrm{Fe} / \mathrm{Mn}$ & 0,28 & 0,196 & 70,4 \\
\hline $\mathbf{B} / \mathbf{N}$ & 0,64 & 0,172 & 27,0 & $\mathrm{Fe} / \mathrm{Zn}$ & 4,98 & 1,229 & 24,7 \\
\hline $\mathbf{B} / \mathbf{P}$ & 10,72 & 2,916 & 27,2 & $\mathbf{M n} / \mathbf{N}$ & 16,36 & 9,928 & 60,7 \\
\hline $\mathbf{B} / \mathbf{K}$ & 0,65 & 0,146 & 22,6 & $\mathbf{M n} / \mathbf{P}$ & 273,98 & 168,76 & 61,6 \\
\hline $\mathbf{B} / \mathbf{C a}$ & 1,86 & 0,877 & 47,2 & $\mathbf{M n} / \mathbf{K}$ & 16,61 & 9,731 & 58,6 \\
\hline B/Mg & 5,34 & 1,900 & 35,6 & $\mathbf{M n} / \mathbf{C a}$ & 44,08 & 28,813 & 65,4 \\
\hline $\mathrm{B} / \mathrm{Cu}$ & 1,99 & 0,692 & 34,8 & $\mathbf{M n} / \mathbf{M g}$ & 135,62 & 94,927 & 70,0 \\
\hline $\mathrm{Fe} / \mathrm{B}$ & 5,32 & 2,931 & 55,1 & $\mathbf{M n} / \mathbf{Z n}$ & 27,96 & 19,554 & 69,9 \\
\hline $\mathbf{M n} / \mathbf{B}$ & 27,51 & 16,621 & 60,4 & $\mathbf{N} / \mathbf{Z n}$ & 1,71 & 0,287 & 16,8 \\
\hline $\mathbf{B} / \mathbf{Z n}$ & 1,11 & 0,402 & 36,2 & $\mathbf{Z n} / \mathbf{P}$ & 10,07 & 1,436 & 14,3 \\
\hline $\mathbf{N} / \mathbf{C u}$ & 3,07 & 0,495 & 16,1 & $\mathbf{Z n} / \mathbf{K}$ & 0,63 & 0,177 & 28,0 \\
\hline $\mathbf{P} / \mathbf{C u}$ & 0,18 & 0,024 & 13,3 & $\mathbf{C a} / \mathbf{Z n}$ & 0,63 & 0,132 & 20,9 \\
\hline $\mathrm{Cu} / \mathrm{K}$ & 0,35 & 0,103 & 29,3 & $\mathrm{Mg} / \mathrm{Zn}$ & 0,21 & 0,031 & 14,9 \\
\hline $\mathbf{C a} / \mathbf{C u}$ & 1,13 & 0,215 & 19,0 & & & & \\
\hline
\end{tabular}

${ }^{(1)} \mathrm{s}=$ desvio-padrão; ${ }^{(2)} \mathrm{CV}=$ coeficiente de variação.

TABELA 6 - Coeficientes de correlação de Pearson $(\mathrm{R})$ entre teores foliares $\left(\mathrm{N}_{\mathrm{F}} . . \mathrm{Zn_{ \textrm {F } }}\right)$ e índices DRIS $\left(\mathrm{N}_{\mathrm{I}} . . \mathrm{Zn} \mathrm{n}_{\mathrm{I}}\right)$, em bananeira do subgrupo Cavendish. Estado de São Paulo, 2007.

\begin{tabular}{|c|c|c|c|c|c|c|c|c|c|c|}
\hline \multirow{2}{*}{ Teor foliar } & \multicolumn{10}{|c|}{ Índice DRIS } \\
\hline & $\mathbf{N}_{\mathbf{I}}$ & $\mathbf{P}_{\mathbf{I}}$ & $\mathbf{K}_{\mathbf{I}}$ & $\mathbf{C a}_{\mathbf{I}}$ & $\mathbf{M g}_{\mathrm{I}}$ & $\mathbf{B}_{\mathbf{I}}$ & $\mathbf{C u}_{\mathbf{I}}$ & $\mathbf{F e}_{\mathbf{I}}$ & $\mathbf{M n}_{\mathrm{I}}$ & $\mathbf{Z n}_{\mathbf{I}}$ \\
\hline \multicolumn{11}{|c|}{ 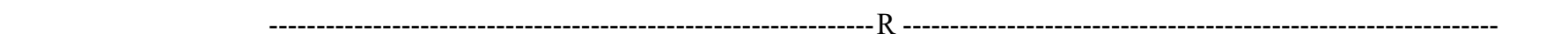 } \\
\hline $\mathbf{N}_{\mathbf{F}}$ & $0,41 * *$ & $-0,15^{*}$ & $0,02 \mathrm{~ns}$ & $-0,16^{*}$ & $-0,06 \mathrm{~ns}$ & $-0,21 * *$ & $0,40 * *$ & $0,19 * *$ & $-0,30 * *$ & $0,06 \mathrm{~ns}$ \\
\hline $\mathbf{P}_{\mathbf{F}}$ & $-0,07 \mathrm{~ns}$ & $0,45^{* *}$ & $0,28 * *$ & $-0,35 * *$ & $-0,35^{* *}$ & $-0,42 * *$ & $0,53 * *$ & $-0,03 \mathrm{~ns}$ & $-0,43 * *$ & $0,41 * *$ \\
\hline $\mathbf{K}_{\mathbf{F}}$ & $-0,01 \mathrm{~ns}$ & $0,42 * *$ & $0,73 * *$ & $-0,69 * *$ & $-0,70^{* *}$ & $-0,43 * *$ & $0,44 * *$ & $-0,03 \mathrm{~ns}$ & $-0,32 * *$ & $0,40 * *$ \\
\hline $\mathrm{Ca}_{\mathrm{F}}$ & $-0,30 * *$ & $-0,59 * *$ & $-0,56^{* *}$ & $0,85^{* *}$ & $0,76^{* *}$ & $0,26 * *$ & $-0,22 * *$ & $0,16^{*}$ & $0,02 \mathrm{~ns}$ & $-0,56^{* *}$ \\
\hline $\mathbf{M g}_{\mathrm{F}}$ & $-0,23 * *$ & $-0,59 * *$ & $-0,53 * *$ & $0,62 * *$ & $0,76^{* *}$ & $0,19 * *$ & $-0,14^{*}$ & $0,28 * *$ & $-0,04 \mathrm{~ns}$ & $-0,46^{* *}$ \\
\hline $\mathbf{B}_{\mathrm{F}}$ & $-0,01 \mathrm{~ns}$ & $-0,36^{* *}$ & $-0,37 * *$ & $0,36 * *$ & $0,45^{* *}$ & $0,87 * *$ & $-0,32 * *$ & $0,14^{*}$ & $0,16^{*}$ & $-0,55^{* *}$ \\
\hline $\mathbf{C u}_{\mathrm{F}}$ & $-0,04 \mathrm{~ns}$ & $-0,01 \mathrm{~ns}$ & $0,06 \mathrm{~ns}$ & $-0,14^{*}$ & $-0,13 n s$ & $-0,40 * *$ & $0,69 * *$ & $0,11 \mathrm{~ns}$ & $-0,31 * *$ & $0,17^{*}$ \\
\hline $\mathbf{F e}_{\mathrm{F}}$ & $-0,20 * *$ & $-0,46^{* *}$ & $-0,22 * *$ & $-0,02 \mathrm{~ns}$ & $0,16^{*}$ & $-0,07 \mathrm{~ns}$ & $-0,06 \mathrm{~ns}$ & $0,85^{* *}$ & $-0,28 * *$ & $0,01 \mathrm{~ns}$ \\
\hline $\mathbf{M n}_{\mathrm{F}}$ & $-0,12 \mathrm{~ns}$ & $-0,32 * *$ & $-0,09 \mathrm{~ns}$ & $0,13 \mathrm{~ns}$ & $0,12 \mathrm{~ns}$ & $-0,13 n s$ & $-0,25 * *$ & $-0,14 \mathrm{~ns}$ & $0,97 * *$ & $-0,31 * *$ \\
\hline $\mathbf{Z n}_{\mathrm{F}}$ & $-0,09 \mathrm{~ns}$ & $0,16^{*}$ & $0,12 \mathrm{~ns}$ & $-0,35 * *$ & $-0,32 * *$ & $-0,49 * *$ & $0,37 * *$ & $0,22 * *$ & $-0,36 * *$ & $0,68 * *$ \\
\hline
\end{tabular}

Significância: $\mathrm{ns}=\mathrm{p}>0,05 ; *=0,05>\mathrm{p}>0,01 \mathrm{e}^{* *}=\mathrm{p}<0,01$ 
TABELA 7 - Equações de regressão múltipla entre teores foliares de N, P, K, Ca, Mg, B, Cu, Fe, Mn e Zn e índices DRIS em bananeira Cavendish. Estado de São Paulo, 2007.

\begin{tabular}{|c|c|c|}
\hline Equação & $\mathbf{R}^{2}$ & Valor $p^{(1)}$ \\
\hline $\mathrm{N}_{\mathrm{F}}=28,52+0,29 \mathrm{~N}_{\mathrm{I}}-0,11 \mathrm{P}_{\mathrm{I}}-0,01 \mathrm{Ca}_{\mathrm{I}}+0,08 \mathrm{Mg}_{\mathrm{I}}-0,11 \mathrm{~B}_{\mathrm{I}}-0,13 \mathrm{Cu}_{\mathrm{I}}+0,04 \mathrm{Fe}_{\mathrm{I}}-0,08 \mathrm{Mn}_{\mathrm{I}}-0,01 \mathrm{Zn}_{\mathrm{I}}$ & 0,48 & $<0,001$ \\
\hline $\mathrm{P}_{\mathrm{F}}=1,69-0,01 \mathrm{~N}_{\mathrm{I}}+0,01 \mathrm{P}_{\mathrm{I}}-0,004 \mathrm{~K}_{\mathrm{I}}-0,005 \mathrm{Ca}_{\mathrm{I}}+0,001 \mathrm{Mg}_{\mathrm{I}}-0,01 \mathrm{~B}_{\mathrm{I}}+0,005 \mathrm{Cu}_{\mathrm{I}}-0,01 \mathrm{Mn}_{\mathrm{I}}-0,004 \mathrm{Zn}_{\mathrm{I}}$ & 0,53 & $<0,001$ \\
\hline $\mathrm{K}_{\mathrm{F}}=27,85-0,13 \mathrm{P}_{\mathrm{I}}+0,25 \mathrm{~K}_{\mathrm{I}}+0,03 \mathrm{Ca}_{\mathrm{I}}+0,08 \mathrm{Mg}_{\mathrm{I}}+0,18 \mathrm{~B}_{\mathrm{I}}+0,22 \mathrm{Cu}_{\mathrm{I}}+0,16 \mathrm{Fe}_{\mathrm{I}}+0,07 \mathrm{Mn}_{\mathrm{I}}+0,11 \mathrm{Zn}_{\mathrm{I}}$ & 0,69 & $<0,001$ \\
\hline $\mathrm{Ca}_{\mathrm{F}}=10,84-0,01 \mathrm{~N}_{\mathrm{I}}-0,06 \mathrm{P}_{\mathrm{I}}+0,04 \mathrm{~K}_{\mathrm{I}}+0,25 \mathrm{Ca}_{\mathrm{I}}+0,06 \mathrm{Mg}_{\mathrm{I}}-0,04 \mathrm{~B}_{\mathrm{I}}+0,09 \mathrm{Cu}_{\mathrm{I}}+0,07 \mathrm{Fe}_{\mathrm{I}}+0,03 \mathrm{Zn}_{\mathrm{I}}$ & 0,87 & $<0,001$ \\
\hline $\mathrm{Mg}_{\mathrm{F}}=3,57-0,01 \mathrm{~N}_{\mathrm{I}}-0,01 \mathrm{P}_{\mathrm{I}}+0,01 \mathrm{~K}_{\mathrm{I}}+0,01 \mathrm{Ca}_{\mathrm{I}}+0,08 \mathrm{Mg}_{\mathrm{I}}-0,01 \mathrm{~B}_{\mathrm{I}}+0,03 \mathrm{Cu}_{\mathrm{I}}+0,02 \mathrm{Fe}_{\mathrm{I}}+0,01 \mathrm{Zn}_{\mathrm{I}}$ & 0,78 & $<0,001$ \\
\hline $\mathrm{B}_{\mathrm{F}}=17,66-0,054 \mathrm{P}_{\mathrm{I}}+0,13 \mathrm{~K}_{\mathrm{I}}+0,02 \mathrm{Ca}_{\mathrm{I}}+0,09 \mathrm{Mg}_{\mathrm{I}}+0,51 \mathrm{~B}_{\mathrm{I}}+0,11 \mathrm{Cu}_{\mathrm{I}}+0,05 \mathrm{Fe}_{\mathrm{I}}+0,03 \mathrm{Mn}_{\mathrm{I}}+0,01 \mathrm{Zn}_{\mathrm{I}}$ & 0,86 & $<0,001$ \\
\hline $\mathrm{Cu}_{\mathrm{F}}=9,51-0,01 \mathrm{~N}_{\mathrm{I}}+0,03 \mathrm{~K}_{\mathrm{I}}+0,05 \mathrm{Ca}_{\mathrm{I}}+0,07 \mathrm{Mg}_{\mathrm{I}}-0,02 \mathrm{~B}_{\mathrm{I}}+0,19 \mathrm{Cu}_{\mathrm{I}}+0,07 \mathrm{Fe}_{\mathrm{I}}+0,02 \mathrm{Mn}_{\mathrm{I}}+0,05 \mathrm{Zn}_{\mathrm{I}}$ & 0,70 & $<0,001$ \\
\hline $\mathrm{Fe}_{\mathrm{F}}=87,29-0,53 \mathrm{~N}_{\mathrm{I}}+0,14 \mathrm{P}_{\mathrm{I}}-0,03 \mathrm{~K}_{\mathrm{I}}+0,37 \mathrm{Ca}_{\mathrm{I}}+0,38 \mathrm{Mg}_{\mathrm{I}}-0,73 \mathrm{~B}_{\mathrm{I}}+0,82 \mathrm{Cu}_{\mathrm{I}}+2,42 \mathrm{Fe}_{\mathrm{I}}-0,09 \mathrm{Mn}_{\mathrm{I}}$ & 0,82 & $<0,001$ \\
\hline $\mathrm{Mn}_{\mathrm{F}}=436,56-3,61 \mathrm{~N}_{\mathrm{I}}-4,79 \mathrm{P}_{\mathrm{I}}-2,80 \mathrm{Ca}_{\mathrm{I}}+1,05 \mathrm{Mg}_{\mathrm{I}}-2,04 \mathrm{~B}_{\mathrm{I}}+1,33 \mathrm{Cu}_{\mathrm{I}}+0,66 \mathrm{Fe}_{\mathrm{I}}+25,20 \mathrm{Mn}_{\mathrm{I}}-1,81 \mathrm{Zn}_{\mathrm{I}}$ & 0,97 & $<0,001$ \\
\hline $\mathrm{Zn}_{\mathrm{F}}=17,30-0,02 \mathrm{P}_{\mathrm{I}}+0,08 \mathrm{~K}_{\mathrm{I}}+0,11 \mathrm{Ca}_{\mathrm{I}}+0,14 \mathrm{Mg}_{\mathrm{I}}-0,005 \mathrm{~B}_{\mathrm{I}}+0,19 \mathrm{Cu}_{\mathrm{I}}+0,12 \mathrm{Fe}_{\mathrm{I}}+0,04 \mathrm{Mn}_{\mathrm{I}}+0,31 \mathrm{Zn}_{\mathrm{I}}$ & 0,71 & $<0,001$ \\
\hline
\end{tabular}

(1) probabilidade de se cometer erro tipo I associada ao teste $\mathrm{F}$ ou nível de significância do teste.

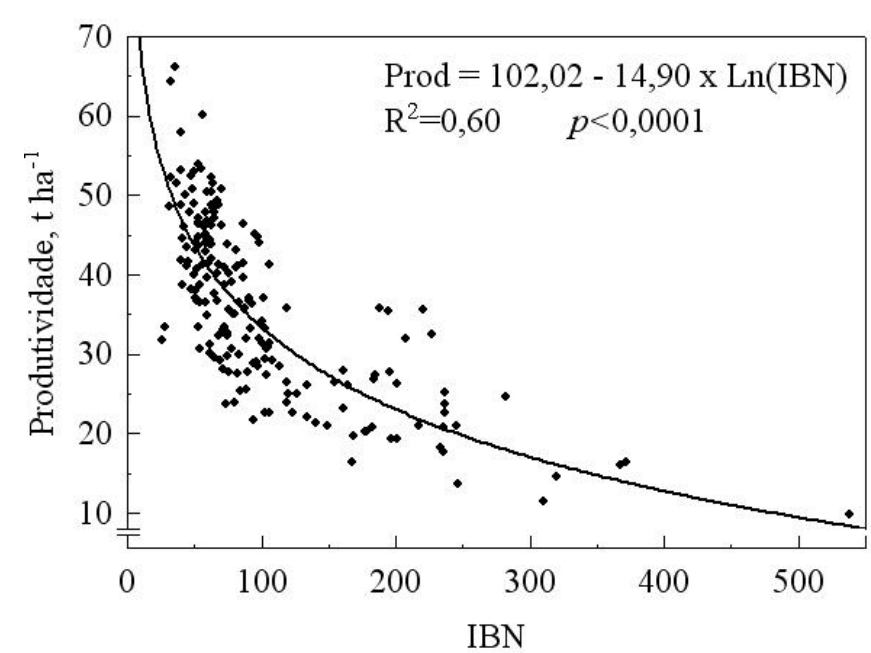

FIGURA 1 - Relação entre o índice de balanço nutricional (IBN) e a produtividade de bananeira do subgrupo Cavendish. Estado de São Paulo, 2007.

\section{CONCLUSÕES}

1 - As relações entre os teores de nutrientes nas folhas, expressas nas normas DRIS, foram eficientes para discriminar as populações de alta e baixa produtividade.

2- A produtividade das bananeiras do subgrupo Cavendish foi associada ao estado nutricional das plantas.

3- O método para derivar níveis críticos de nutrientes no tecido foliar, empregando-se o DRIS, foi eficiente para bananeira.

4 - As normas DRIS obtidas no presente trabalho, para as bananeiras do subgrupo Cavendish, necessitam de validação antes do seu emprego na diagnose nutricional.

\section{AGRADECIMENTOS}

Ao Pesquisador Ondino Cleante Bataglia, pelas sugestões.

\section{REFERÊNCIAS}

ANGELES, D.E.; SUMNER, M.E.; LAHAV, E. Preliminary DRIS norms for banana. Journal of Plant Nutrition, New York, v. 16, p.1059-70, 1993.

BATAGLIA, O.C.; FURLANI, A.M.C.; TEIXEIRA, J.P.F.; FURLANI, P.R.; GALLO, J.R. Métodos de análise química de plantas. Campinas: IAC, 1983.48p. (Boletim Técnico, 78).

BATAGLIA, O.C.; QUAGGIO, J.A.; SANTOS, W.R.; ABREU, M.F. Diagnose nutricional do cafeeiro pelo DRIS variando-se a constante de sensibilidade dos nutrientes de acordo com a intensidade e freqüência de respostas na produção. Bragantia, v. 63 , p. 253-263, 2004.

BATAGLIA, O.C.; SANTOS, W.R. Efeito do procedimento de cálculo e da população de referência nos índices do sistema integrado de diagnose e recomendação (DRIS). Revista Brasileira de Ciência do Solo, Campinas, v.14, p.339-344, 1990.

BEAUFILS, E.R. Diagnosis and recommendation integrated system (DRIS). South Africa: University of Natal, 1973. 132p. (Soil Science Bull., 1).

BEVERLY, R.B.; HALLMARK, W.B. Prescient diagnostic analysis: a proposed new approach to evaluating plant nutrient diagnostic methods. Communications in Soil Science and Plant Analysis, New York, v. 23, p.2633-40, 1992. 
DAMATTO JR., E.R. Efeitos da adubação com composto orgânico na fertilidade do solo, desenvolvimento, produção e qualidade de frutos de bananeira 'Prata-anã' (Musa AAB)., 2005. $70 \mathrm{f}$. Dissertação (Mestrado em Agronomia) - Faculdade de Ciências Agronômicas, Universidade Estadual Paulista, Botucatu, 2005.

IBGE. Instituto Brasileiro de Geografia e Estatística. Disponível em: $<$ http://www.ibge.gov.br $>$. Acesso em: 30 out. 2006.

JONES, C.A. Proposed modifications of the diagnosis and recommendation integrated system (DRIS) for interpreting plant analyses. Communications in Soil Science and Plant Analysis, New York, v.12, p.785-94, 1981.

JOSEPH, M.; J.; RANGANATHAN, C.R. Diagnosis and recommendation integrated system: 2 . derivation of critical level of leaf nutrient concentration in rubber. Indian Journal of Natural Rubber Research, Kottayam, v.9, n.1, p.17-21, 1996.

LAHAV, E. Banana nutrition. In: GOWEN, S. (Ed.) Bananas and plantains. London: Chapman \& Hall, 1995. p.258-316.

LAHAV, E.; TURNER, D.W. Temperature influences the composition of diagnostic samples used to assess the nutrient status of banana plants. Scientia Horticulturae, Amsterdam, v.27, p. $275-83,1985$.

MARTIN-PRÉVEL, P. Bananier. In: MARTIN-PRÉVEL, P.; GAGNARD, J.; GAUTIER, P. (Eds.) L'analyse végétale dans le contrôle de l'alimentation des plantes tempérées et tropicales. Paris: Tec\&Doc, 1984. p.715-51.

MARTIN-PRÉVEL, P. Echantillonnage du bananier pour l'analyse foliaire: Conséquences des différences de techniques. Fruits, Paris, v.32, p.151-66, 1977.

MARTIN-PRÉVEL, P. Past, present and future of tropical fruit nutrition with special reference to banana nutrition. Acta Horticulturae, Leuven, v.275, p. 523-34, 1990.

MOURÃO FILHO, F.A.A. DRIS: concepts and applications on nutritional diagnosis in fruit crops. Scientia Agricola, Piracicaba, v.61, n.5, p.550-560, 2004.

NEEDHAM, T.D.; BURGER, J.A.; ODERWALD, R.G. Relationship between diagnosis and recommendation integrated system (DRIS) optima and foliar nutrient critical levels. Soil Science Society of America Journal, Madison, v.54, p.883-886, 1990.

QUAGGIO, J.A.; RAIJ, B. van. Frutíferas. In: RAIJ, B. van et al. (Eds.) Recomendações de adubação e calagem para o Estado de São Paulo. 2.ed.rev. Campinas: IAC, 1997. p.121-153 (Boletim Técnico, 100)
REIS JÚNIOR, R.A.; MONNERAT, P.H. Norms establishment of the Diagnosis and Recommendation Integrated System (DRIS) for nutritonal diagnosis of sugarcane. Pesquisa Agropecuária Brasileira, Brasília, v. 38, p. 277-282, 2003.

SILVA, E.B.; NOGUEIRA, F.D.; GUIMARÃES, P.T.G. Uso do DRIS na avaliação do estado nutricional do cafeeiro em resposta à adubação potássica. Revista Brasileira de Ciência do Solo, Viçosa, v. 27, p.247-255, 2003.

SILVA, E.B.; RODRIGUES, M.G.V. Levantamento nutricional dos bananais da região norte de Minas Gerais. Revista Brasileira de Fruticultura, Jaboticabal, v. 23, n. 3, p. 695-698, 2001.

SILVA, J.T.A. Avaliação nutricional da bananeira Prata Anã (AAB) sob irrigação, no semi-árido do Norte de Minas Gerais. 2004. 129 f. Tese (Doutorado em Solos e Nutrição de Plantas) - Universidade Federal de Lavras, Lavras, 2004.

SILVA, J.T.A.; CARVALHO, J.G. Avaliação nutricional da bananeira Prata Anã (AAB), sob irrigação, no semi-árido do Norte de Minas Gerais, pelo método DRIS. Ciência e Agrotecnologia, Lavras, v.29, n. 4, p.731-739, 2005.

TEIXEIRA, L.A.J.; NATALE, W.; RUGGIERO, C. Nitrogen and potassium fertilization of 'Nanicão' banana (Musa AAA Cavendish subgroup) under irrigated and non-irrigated conditions. Acta Horticulturae, Leuven, v.275, p.771-9, 2002a.

TEIXEIRA, L.A.J.; NATALE, W.; MARTINS, A.L.M. Nitrogênio e potássio via fertirrigação e adubação convencional-estado nutricional das bananeiras e produção de frutos. Revista Brasileira de Fruticultura, Jaboticabal, v.29, n.1, p.153-160, 2007.

TEIXEIRA, L.A.J.; SANTOS, W. R.; BATAGLIA, O. C. Diagnose nutricional para nitrogênio e potássio em bananeira por meio do Sistema Integrado de Diagnose e Recomendação (DRIS) e de níveis críticos. Revista Brasileira de Fruticultura, Jaboticabal, v.24, n.2, p.530-535, 2002b.

TURNER, D.W.; LAHAV, E. Temperature influences nutrient absorption and uptake rates of bananas grown in controlled environments. Scientia Horticulturae, Amsterdam, v.26, p.31122,1985 .

WALWORTH, J.L.; SUMNER, M.E. The diagnosis and recommendation integrated system (DRIS). Advances in Soil Science, New York, v.6, p.149-88, 1987. 